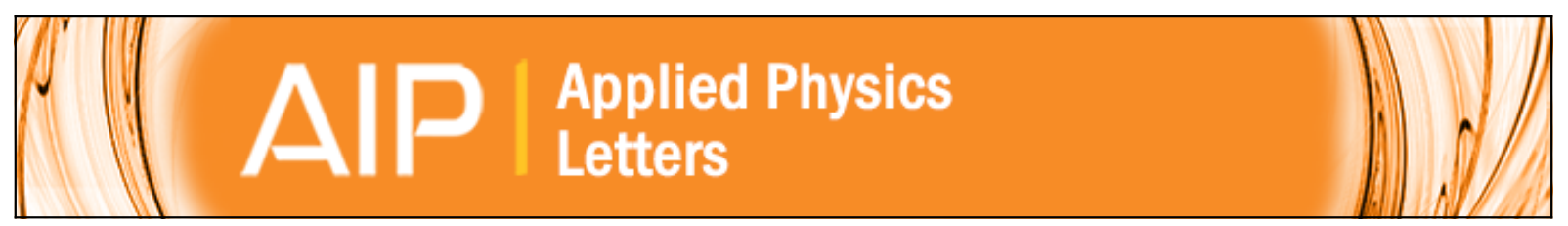

\title{
An ion-gated bipolar amplifier for ion sensing with enhanced signal and improved noise performance
}

Da Zhang, Xindong Gao, Si Chen, Hans Norström, Ulf Smith, Paul Solomon, Shi-Li Zhang, and Zhen Zhang

Citation: Applied Physics Letters 105, 082102 (2014); doi: 10.1063/1.4894240

View online: http://dx.doi.org/10.1063/1.4894240

View Table of Contents: http://scitation.aip.org/content/aip/journal/apl/105/8?ver=pdfcov

Published by the AIP Publishing

\section{Articles you may be interested in}

Large area graphene ion sensitive field effect transistors with tantalum pentoxide sensing layers for $\mathrm{pH}$ measurement at the Nernstian limit

Appl. Phys. Lett. 105, 083101 (2014); 10.1063/1.4894078

Hydrogen ion-selective electrolyte-gated organic field-effect transistor for $\mathrm{pH}$ sensing

Appl. Phys. Lett. 104, 193305 (2014); 10.1063/1.4878539

Nanodiamond-gated silicon ion-sensitive field effect transistor

Appl. Phys. Lett. 98, 153507 (2011); 10.1063/1.3568887

Nanodiamond-gated diamond field-effect transistor for chemical sensing using hydrogen-induced transfer doping for channel formation

Appl. Phys. Lett. 97, 203503 (2010); 10.1063/1.3518060

Theoretical study of electrolyte gate Al Ga N Ga N field effect transistors

J. Appl. Phys. 97, 033703 (2005); 10.1063/1.1847730

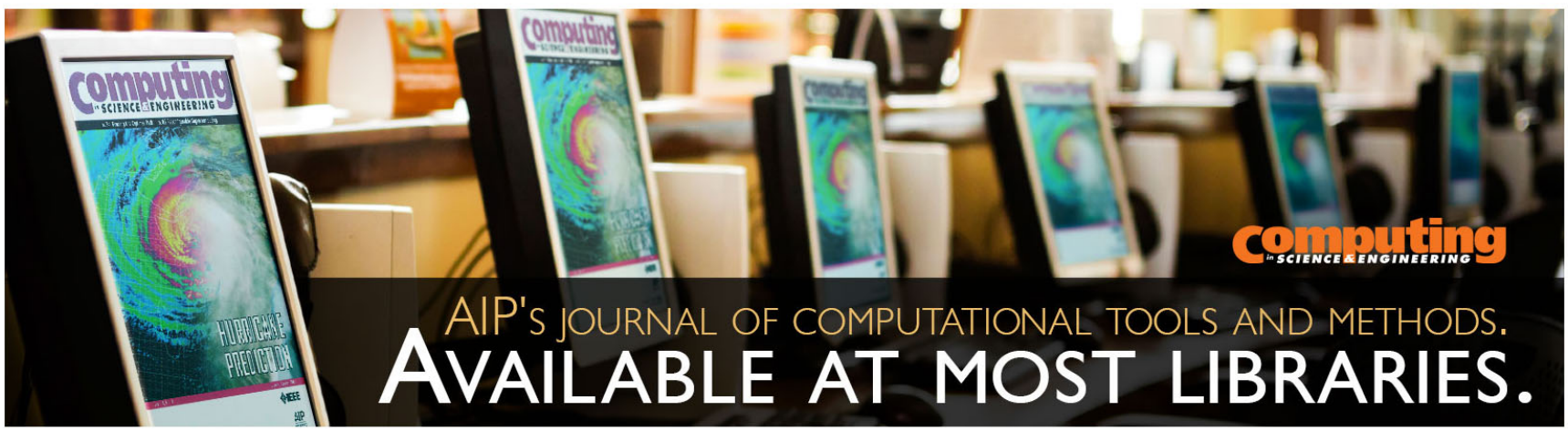




\title{
An ion-gated bipolar amplifier for ion sensing with enhanced signal and improved noise performance
}

\author{
Da Zhang, Xindong Gao, Si Chen, ${ }^{\text {a) }}$ Hans Norström, Ulf Smith, Paul Solomon, ${ }^{\text {b) }}$ \\ Shi-Li Zhang, and Zhen Zhang ${ }^{\mathrm{c})}$ \\ Solid-State Electronics, The Angström Laboratory, Uppsala University, SE-751 21 Uppsala, Sweden
}

(Received 30 July 2014; accepted 16 August 2014; published online 25 August 2014)

\begin{abstract}
This work presents a proof-of-concept ion-sensitive device operating in electrolytes. The device, i.e., an ion-gated bipolar amplifier (IGBA), consists of a modified ion-sensitive field-effect transistor (ISFET) intimately integrated with a vertical bipolar junction transistor for immediate current amplification without introducing additional noise. With the current non-optimized design, the IGBA is already characterized by a 70-fold internal amplification of the ISFET output signal. This signal amplification is retained when the IGBA is used for monitoring $p \mathrm{H}$ variations. The tight integration significantly suppresses the interference of the IGBA signal by external noise, which leads to an improvement in signal-to-noise performance compared to its ISFET reference. The IGBA concept is especially suitable for biochips with millions of electric sensors that are connected to peripheral readout circuitry via extensive metallization which may in turn invite external interferences leading to contamination of the signal before it reaches the first external amplification stage. (C) 2014 AIP Publishing LLC. [http://dx.doi.org/10.1063/1.4894240]
\end{abstract}

Electronic sensing of ions in electrolytes using ion sensitive field-effect transistor (ISFET) technology ${ }^{1}$ finds a vast variety of applications in chemistry and biomedicine. ${ }^{2}$ The recent commercialization of an ISFET-based biochip for semiconductor genome sequencing ${ }^{3}$ and demonstration of an ISFET-based on-chip quantitative polymerase chain reaction $(\mathrm{qPCR})^{4}$ represent potential transistor-based revolutions in healthcare. Both techniques employ standard complementary metal-oxide-semiconductor (CMOS) fabrication technology and ingeniously build on correlating the $p \mathrm{H}$ variation in electrolytes with proton release upon the chemical binding of probing nucleotides with target DNA strands. However, the insufficient signal-to-noise performance with ordinary ISFETs operating in electrolytes requires the use of a large number of identical DNA copies (up to $10^{6}$ ) per transistor sensor in the sequencing chip ${ }^{3}$ in order to generate reliable signals. Producing such a large number of DNA copies increases not only sample preparation complexity but also molecular error rates. In order to fully exploit CMOS technology for high throughput, these biochips integrate ${ }^{3,4}$ a large number of sensors that are administrated by external readout circuitry including signal amplification via extensive metallization. Apart from contributing thermal and lowfrequency noise, the long metal wiring is subject to lowfrequency noise coupling which can interfere with biomedical signals that mostly span in the same frequency domain. ${ }^{5}$ Indeed, gate coupling to metallization contacts in an ISFET has been shown to generate low-frequency noise that is up to two orders of magnitude higher than what gate coupling to the ISFET channel produces. ${ }^{6}$ Hence, it is highly desirable to

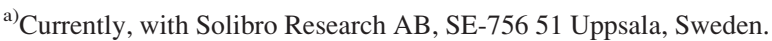

b) IBM T. J. Watson Research Center, Yorktown Heights, New York 10598, USA.

c) Author to whom correspondence should be addressed. Electronic mail: zhen.zhang@angstrom.uu.se.
}

develop new device concepts that can yield strong signal and enhance signal-to-noise performance by avoiding generation of extra internal noise as well as by suppressing interference of external noise. This letter presents such an approach represented by the ion-gated bipolar amplifier (IGBA) concept. An IGBA is structurally similar to a lateral insulated-gate bipolar transistor (LIGBT). ${ }^{7}$ But the IGBA assumes different designs regarding geometry and doping, including elimination of the long lateral drift region needed in the LIGBT. Thus, the IGBA consists of a high-gain bipolar junction transistor (BJT) intimately integrated with a metal-oxide-semiconductor field-effect transistor (MOSFET). Unlike LIGBT for high-power switching applications, the IGBA focuses on high internal amplification of the MOSFET output signal without running into latch-up. Since BJT is known to generate negligible low-frequency noise ${ }^{8}$ in contrast to the surface device MOSFET, our IGBA is of significant potential as an advanced sensor with large signal-to-noise ratio (SNR) which is especially attractive for high-density sensor arrays integrated via extensive metallization. ${ }^{3,4}$ With significantly amplified signal and improved low-frequency noise performance, the IGBA operating in electrolytes is further demonstrated in the present work by $p \mathrm{H}$ measurement with the MOSFET acting as the sensing interface, i.e., ISFET with an extended gate (EG). ${ }^{9}$

The cross section of our IGBA is schematically shown in Fig. 1 (left), with its MOSFET resembling, to a large extent, the structure of a laterally diffused MOSFET (LDMOS) without $n+$ contact at the right. The device was fabricated on a $150-\mathrm{mm}$ Si (100) wafer with a lightly doped epitaxial layer grown on a heavily doped substrate. It comprises four accessible terminals: emitter $E$, collector $C$, source $S$, and gate $G$. All junctions were formed using ion implantation followed by heat treatments in accordance to extensive simulations. Briefly, the $p+$ substrate serves as the collector of a vertical pnp $\mathrm{BJT}\left(T_{1}\right)$. The $n$ region acts not 


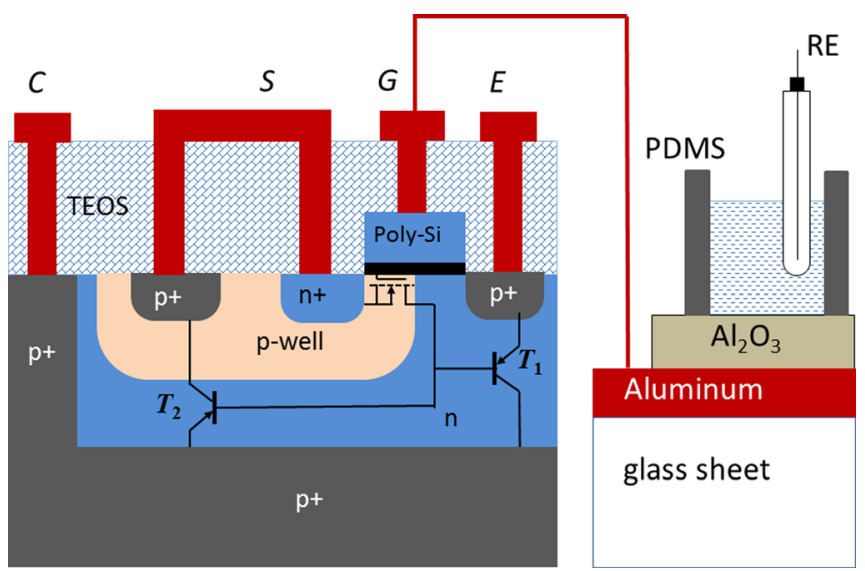

FIG. 1. Schematic cross section of our IGBA, as well as the EG-based $p H$ measurement setup. The designed length and width of the poly-Si gate are $10 \mu \mathrm{m}$ and $20 \mu \mathrm{m}$, respectively.

only as the base in the BJT but also as the drift region in $n$ channel ISFET. Thus, the drain current, $I_{\mathrm{D}}$, of the ISFET is immediately amplified by $T_{1}$ and leaves the device as a significantly enhanced collector current, $I_{C}$. As the gate leakage was found to be negligible and the current gain of the vertical pnp BJT was large (see later), the source current is represented by $I_{\mathrm{D}}$ and the emitter current approximated by $I_{\mathrm{C}}$ in the reminder of this work. Electrical characterization was carried out using an HP4155 precision semiconductor analyzer. During the measurement, both the source and collector terminals were biased at $0 \mathrm{~V}$ with respective to the common ground. The emitter terminal, i.e., the power supply of the IGBA, was biased above the built-in potential of the $p+-n$ emitter-base junction to turn on the device. For lowfrequency noise characterization, the IGBA and its internal ISFET were analyzed separately. In both measurements, $V_{\mathrm{G}}$ was identical and so was $V_{\mathrm{S}}$ (grounded). $I_{\mathrm{C}}$ (i.e., $I_{\mathrm{E}}$ ) of the IGBA was monitored at a certain $V_{\mathrm{E}}$, while $V_{C}$ was grounded. $I_{\mathrm{D}}$ of the ISFET was accessed via an additional contact to its drain terminal with a bias $V_{D}$ (not shown in Fig. 1) while leaving terminals $C$ and $E$ unconnected. In order to make a fair comparison, $V_{\mathrm{D}}$ for the ISFET was set to yield $I_{\mathrm{D}}$ identical to $I_{\mathrm{S}}$ of the IGBA. The biases in the terminals were applied using batteries. The fluctuation in $I_{\mathrm{D}}$ and $I_{\mathrm{C}}$ was first amplified by a TI TL071 low-noise preamplifier and then monitored at a sampling rate of $1000 \mathrm{~Hz}$ for $20 \mathrm{~s}$ using an Agilent B1530A Waveform Generator/Fast Measurement Unit. The noise spectrum was analyzed using the signal processing toolbox in Matlab.
In the demonstration setup for $p \mathrm{H}$ measurement in electrolytes, an additional $\mathrm{EG}^{9}$ was employed and is also schematically shown in Fig. 1 (right). A 1- $\mu$ m-thick Al layer, which was sputter-deposited on a glass sheet, is directly connected to the gate electrode of the IGBA during $p \mathrm{H}$ sensing. A $100-n m ~_{2} \mathrm{O}_{3}$ layer, which was grown by means of atomic layer deposition, acts as the $p \mathrm{H}$ sensitive membrane ${ }^{2}$ and electrically separates the Al layer from the electrolyte. A polydimethylsiloxane (PDMS) container was placed on top of the $\mathrm{Al}_{2} \mathrm{O}_{3} / \mathrm{Al}$ layer stack for the electrolyte to be analyzed in the $p \mathrm{H}$ measurement experiment. To this end, an $\mathrm{AgCl} / \mathrm{Ag}$ reference electrode (RE) immersed in the electrolyte ${ }^{10}$ was set at a potential denoted as $V_{\mathrm{RE}}$. The gate electrode then assumed a certain potential denoted as $V_{\mathrm{G}}$ via the electrolyte. In our sampling experiment, current variations of the IGBA at a constant $V_{\mathrm{RE}}$ were monitored when the $p \mathrm{H}$ value was altered.

The transfer characteristics of the IGBA were first analyzed and the results are depicted in Fig. 2(a), with $I_{\mathrm{D}}$ to represent the internal ISFET, $I_{\mathrm{C}}$ the IGBA, and $g_{\mathrm{mD}}$ and $g_{\mathrm{mC}}$ the corresponding transconductances. The threshold voltage, $V_{\mathrm{T}}$, and the bias point $V_{0}$ at which the peak $g_{\mathrm{m}}$ is reached are indicted in the figure with vertical broken lines. Here, the gate was directly set at $V_{\mathrm{G}}$. In Fig. 2(b), the current gain of the IGBA over the ISFET itself, i.e., $I_{\mathrm{C}} / I_{\mathrm{D}}$, is shown. At an emitter bias $V_{\mathrm{E}}=0.75 \mathrm{~V}$, a fairly large DC current gain $I_{\mathrm{C}} /$ $I_{\mathrm{D}}=60-80$ is readily obtained in both regions of operation, i.e., weak inversion (subthreshold) (below $V_{\mathrm{T}}$ ) and moderate inversion (above $V_{\mathrm{T}}$ ). ${ }^{11}$ The increased current drive of the IGBA compared to ISFET will also give shorter signal rise times hence higher sensing speeds, which is especially valuable for detection of transient signals from small sensing areas or volumes.

The output characteristics, $I_{\mathrm{C}}$ and $I_{\mathrm{D}}$ versus $V_{\mathrm{E}}$, of the device were also evaluated and the results are shown in Fig. 3, (a) for $I_{\mathrm{C}}-V_{\mathrm{E}}$ and (b) for $I_{\mathrm{D}}-V_{\mathrm{E}}$ at different $V_{\mathrm{G}}$. It is noticed that the $I_{\mathrm{C}} / I_{\mathrm{D}}$ ratio tends to decrease with increasing $V_{\mathrm{E}}$. This decrease is caused by the onset of the parasitic BJT $T_{2}$ and/or the current leakage laterally from the $n$-base into the $p$-well region (cf. Fig. 1). As a result, $T_{2}$ and the leakage shunt part of $I_{\mathrm{C}}$ that should otherwise have flowed into the collector of the vertical pnp BJT $T_{1}$. This in turn leads to the recorded decrease in output $I_{C}$ and thus in $I_{\mathrm{C}} / I_{\mathrm{D}}$ ratio. However, the device did not enter the latch-up region since the characteristics could be restored once $V_{\mathrm{E}}$ was reduced. Further design improvements are needed to reduce such parasitic effects. Besides, $T_{1}$ as the amplifier of the IGBA does
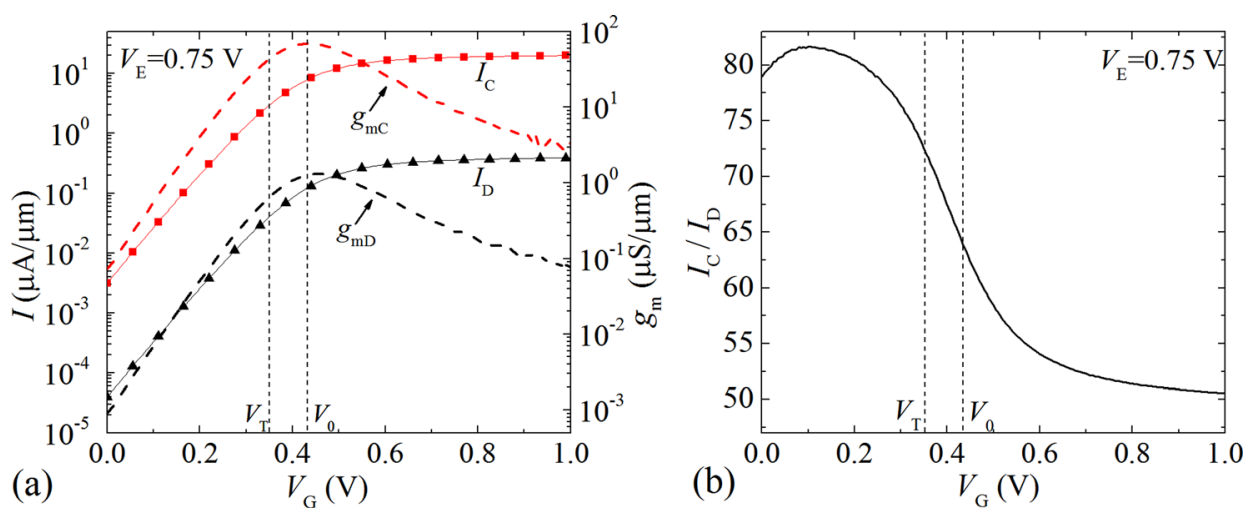

FIG. 2. (a) $I_{\mathrm{C}}-V_{\mathrm{G}}$ transfer and $g_{\mathrm{mC}}$ characteristics of the IGBA compared to $I_{\mathrm{D}}-V_{\mathrm{G}}$ and $g_{\mathrm{mD}}$ of its reference ISFET and (b) current gain, defined as $I_{\mathrm{C}} / I_{\mathrm{D}}$, versus $V_{\mathrm{G}}$ showing a high and fairly constant gain in both the subthreshold region below $V_{\mathrm{T}}=0.35 \mathrm{~V}$ and the moderate inversion region above $V_{\mathrm{T}}=0.35 \mathrm{~V}$.

$V_{\mathrm{G}}(\mathrm{V})$



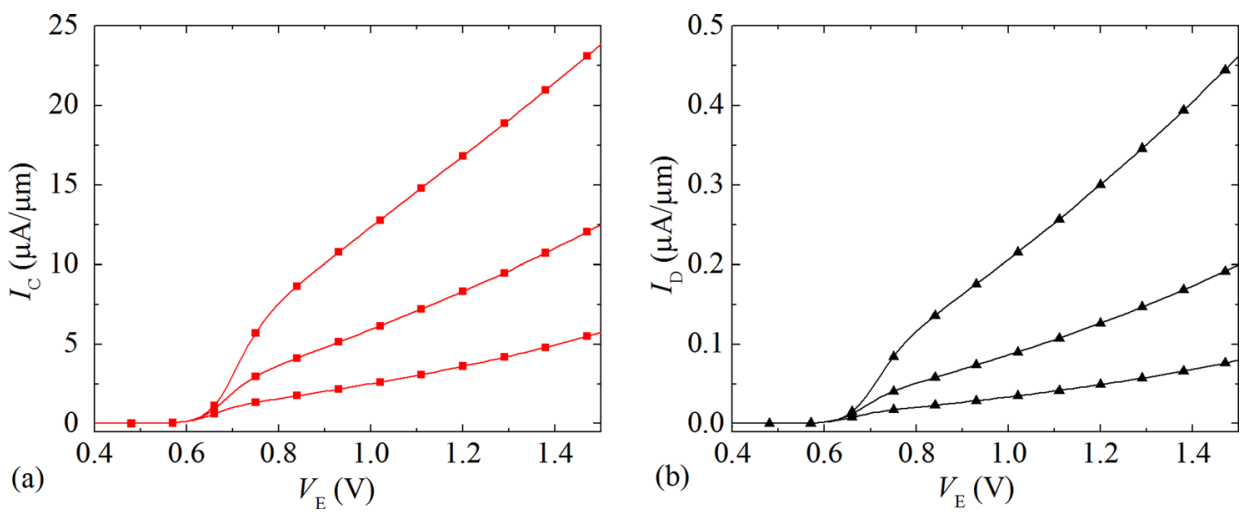

FIG. 3. Output characteristics (a) $I_{\mathrm{C}^{-}}$ $V_{\mathrm{E}}$ of the IGBA compared to (b) $I_{\mathrm{D}^{-}} V_{\mathrm{E}}$ of its reference ISFET. For both cases, $V_{\mathrm{G}}$ is set to $0.3 \mathrm{~V}, 0.35 \mathrm{~V}$, and $0.4 \mathrm{~V}$ from bottom to top. Note the difference in scale of the vertical axes. not interact with the internal LDMOS to render a thyristor. The lateral thyristor can thus, in principle, be readily disabled without deteriorating the current gain.

The noise on $I_{\mathrm{D}}$ is inevitably also amplified by the internal BJT. To quantify noise and SNR, the power spectrum density of current noise, $S_{\mathrm{I}}$, as a function of frequency, $f$, was first characterized for $I_{\mathrm{C}}$ of the IGBA, $S_{\mathrm{I}}^{\mathrm{IGBA}}$, and $I_{\mathrm{D}}$ of the internal ISFET, $S_{\mathrm{I}}^{\text {ISFET }}$. The $V_{\mathrm{G}}$-referred power spectrum density of voltage noise, $S_{\mathrm{V}}$, was then obtained by employing the relationship $S_{\mathrm{V}}=S_{\mathrm{I}} / g_{\mathrm{m}}{ }^{2}$. The resultant $S_{\mathrm{V}}{ }^{\text {IGBA }}$ and $S_{\mathrm{V}}{ }^{\text {ISFET }}$ measured at $V_{\mathrm{G}}=0.25 \mathrm{~V}$ and $V_{\mathrm{E}}=0.75 \mathrm{~V}$ in the subthreshold regime with relatively low $I_{\mathrm{C}}$ and $I_{\mathrm{D}}$ are depicted in Fig. 4(a). The dashed line shows the calculated thermal noise of the ISFET under the same bias conditions using $S_{\mathrm{V}}=4 k T \frac{2}{3} \frac{1}{g_{\mathrm{mD}}} .{ }^{12}$ Two important observations can be made. First, the $S_{\mathrm{V}}{ }^{\text {IGBA }}$ curve is almost 10 times lower than the $S_{\mathrm{V}}{ }^{\text {ISFET }}$ curve in the entire measurement range from $f=1 \mathrm{~Hz}$ to $f=500 \mathrm{~Hz}$. Second, the large spikes at $50 \mathrm{~Hz}$ and its higher harmonics in the ISFET are completely suppressed in the IGBA since they are added at the IGBA output where signal levels are stronger.

This much desired advantage with improved noise performance of the IGBA primarily results from signal amplification before it becomes contaminated by external interferences including noise generated by the low-noise preamplifier in the characterization system. As expected, the difference between $S_{\mathrm{V}}{ }^{\text {IGBA }}$ and $S_{\mathrm{V}}{ }^{\text {ISFET }}$ diminishes when $V_{\mathrm{G}}$ is increased above $V_{\mathrm{T}}$ at which both $I_{\mathrm{C}}$ and $I_{\mathrm{D}}$ increase by more than 100 times and the external interferences are less influential. Hence, the gain in SNR for the IGBA with referenced to the ISFET, which is calculated with Gain SNR $=\sqrt{\frac{g_{\mathrm{mC}}^{2}}{\int S_{\mathrm{I}}^{\mathrm{IGBA}} \mathrm{d} f}} / \sqrt{\frac{g_{\mathrm{mD}}^{2}}{\int S_{\mathrm{I}}^{\mathrm{ISFET}} \mathrm{d} f}}=\sqrt{\frac{\int S_{\mathrm{V}}^{\mathrm{ISFET}} \mathrm{d} f}{\int S_{\mathrm{V}}^{\mathrm{IGBA}} \mathrm{d} f}}$, asymptotically approaches unity with increasing $V_{\mathrm{G}}$, cf.
Fig. 4(b). The remaining SNR-benefit for the IGBA at $V_{\mathrm{G}} \geq V_{\mathrm{T}}$ is simply a consequence of the effective suppression of the spikes associated with $50 \mathrm{~Hz}$ and its harmonics. The effect of the spikes on Gain_SNR is better elucidated by including them in the $S_{\text {I }}$ integral from $f=1 \mathrm{~Hz}$ to $f=500 \mathrm{~Hz}$; Gain_SNR is found to increase from 2 to 3 if the integration spans from $f=1 \mathrm{~Hz}$ to $f=50 \mathrm{~Hz}$ to around 6 when the integration extends to $f=500 \mathrm{~Hz}$. The results in Fig. 4 also confirm that the internal BJT does not generate measurable additional low-frequency noise. Therefore, such a solution with a high output current and a large noise margin that is offered by the IGBA is especially important for driving the long and closely packed wordlines and bitlines present in a genome sequencing chip ${ }^{3}$ or for in-vivo medical testing in noisy electrical environments.

The IGBA is structurally similar to a conventional LIGBT used for high-power $(\mathrm{kW} / \mathrm{kV})$ switching of hightension electrical power-lines. ${ }^{13}$ However, it should be noted that both the basic function and the operating conditions are totally different for the IGBA that is designed as a gate-viaelectrolyte device with large readout signal and suppressed noise in response to surface charge variations, and for lowvoltage operation. These are operating conditions totally out of scope for the high-power LIGBTs. The absence of the long lateral drift region in the IGBA not only eliminates a potential noise source but also is advantageous for highdensity integration in biochips desired for high-throughput data generation. ${ }^{3,4}$ So far, only a gated lateral BJT connected in parallel with a classical ISFET, able to operate in a hybrid mode with active amplification, has been reported for sensor applications. ${ }^{14} \mathrm{~A}$ drawback with such a parallel arrangement of the two devices is the inherently low gain of the BJT. By contrast, the vertical BJT in our IGBA structure allows much higher current gains to be attained.
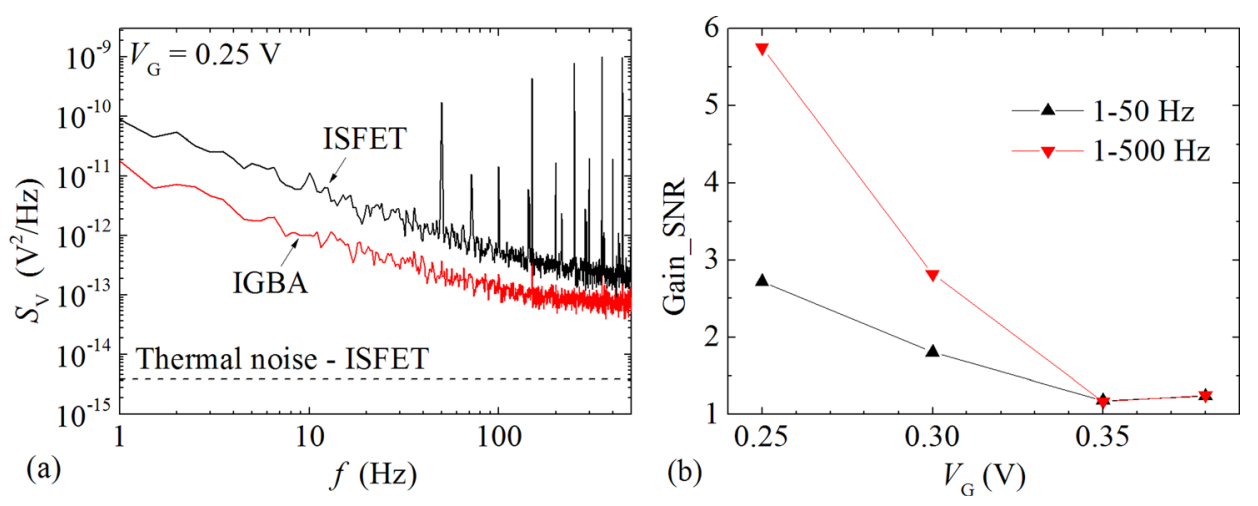

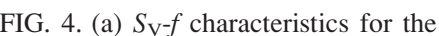
IGBA and its ISFET counterpart, at $V_{\mathrm{E}}=0.75 \mathrm{~V}$ and $V_{\mathrm{G}}=0.25 \mathrm{~V}$, with the dashed line showing the calculated thermal noise of the ISFET under the same bias conditions. (b) Gain_SNR at different $V_{\mathrm{G}}$ for two frequency integration intervals in (a). 


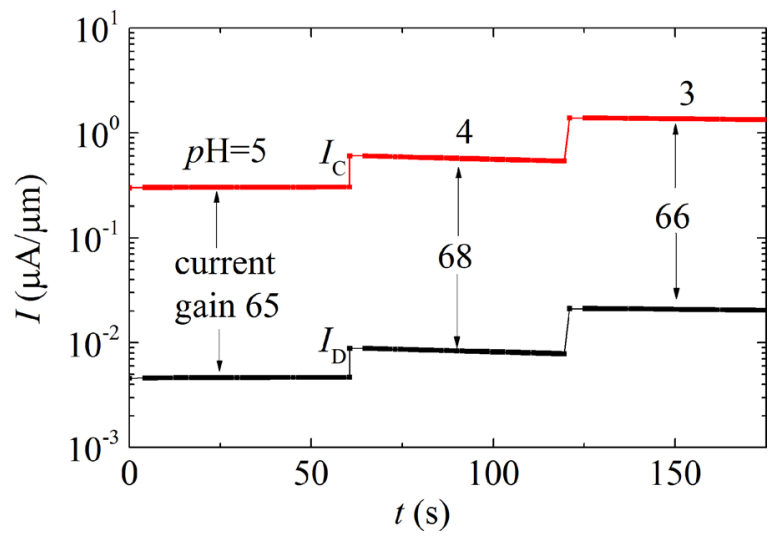

FIG. 5. $I_{\mathrm{C}^{-} t}$ measurement for $p \mathrm{H}$ sensing with the IGBA compared to $I_{\mathrm{D}^{-}} t$ of its reference ISFET. The RE was biased at $1.5 \mathrm{~V}$, i.e., $V_{\mathrm{RE}}=1.5 \mathrm{~V}$, to ensure the operation of the device around $V_{0}$. The $p \mathrm{H}$ values are nominal.

Finally, our experiment with electrolytes in the form of monitoring $I_{\mathrm{D}}$ and $I_{\mathrm{C}}$ variations with time, $t$, was carried out by operating the IGBA at $V_{\mathrm{RE}}=1.5 \mathrm{~V}$ and $V_{\mathrm{E}}=0.7 \mathrm{~V}$. The results are shown in Fig. 5 for $p \mathrm{H}$ values from 5 to 4 and then to 3 . Very large changes in $I_{\mathrm{C}}$ from $0.3-0.6 \mu \mathrm{A} / \mu \mathrm{m}$ to $0.6-1.2 \mu \mathrm{A} / \mu \mathrm{m}$ are obtained. The $p \mathrm{H}$ values are nominal. They were altered manually by the titration of hydrogen chloride ( $\mathrm{HCl}$ ) into the solution. The results in Fig. 5 clearly demonstrate the performance advantages of the IGBA in $p \mathrm{H}$ sensing applications.

In conclusion, we have demonstrated the application of our IGBA as an advanced ion sensor by characterizing it with $p \mathrm{H}$ measurements. The intimate integration of a MOSFET/ISFET with a low-noise vertical BJT for immediate and direct current amplification yields a 70 -fold intrinsic current gain as well as clearly demonstrated advantages in noise performance compared to the ISFET alone. The high current gain also applies when the device is used for $p \mathrm{H}$ sensing. These characteristics make the IGBA-based ion sensor especially attractive in fields such as genome sequencing which require large-scale, densely integrated ion sensors necessary for promptly obtaining huge quantities of data. In such applications, an immediate pre-amplification is vital for eliminating the possibility of contaminating the sensing signal by additional noise along the signal path to the first external amplification stage.

The authors are grateful to Kristina Wiberg for assistance in device processing and to Dr. Klas-Håkan Eklund for fruitful discussions. This work was supported by Swedish Foundation for Strategic Research (SSF) through an Ingvar Carlsson Award (Z.Z.), Uppsala University with a starting grant for the chair professorship (S.-L.Z.), and China Scholarship Council via a scholarship (D.Z.).

${ }^{1}$ P. Bergveld, IEEE Trans. Biomed. Eng. BME-17, 70 (1970).

${ }^{2}$ P. Bergveld, Sens. Actuators, B 88, 1 (2003).

${ }^{3}$ J. M. Rothberg, W. Hinz, T. M. Rearick, J. Schultz, W. Mileski, M. Davey, J. H. Leamon, K. Johnson, M. J. Milgrew, M. Edwards, J. Hoon, J. F. Simons, D. Marran, J. W. Myers, J. F. Davidson, A. Branting, J. R. Nobile, B. P. Puc, D. Light, T. A. Clark, M. Huber, J. T. Branciforte, I. B. Stoner, S. E. Cawley, M. Lyons, Y. Fu, N. Homer, M. Sedova, X. Miao, B. Reed, J. Sabina, E. Feierstein, M. Schorn, M. Alanjary, E. Dimalanta, D. Dressman, R. Kasinskas, T. Sokolsky, J. A. Fidanza, E. Namsaraev, K. J. McKernan, A. Williams, G. T. Roth, and J. Bustillo, Nature 475, 348 (2011).

${ }^{4}$ C. Toumazou, L. M. Shepherd, S. C. Reed, G. I. Chen, A. Patel, D. M. Garner, C.-J. A. Wang, C.-P. Ou, K. Amin-Desai, P. Athanasiou, H. Bai, I. M. Q. Brizido, B. Caldwell, D. Coomber-Alford, P. Georgiou, K. S. Jordan, J. C. Joyce, M. La Mura, D. Morley, S. Sathyavruthan, S. Temelso, R. E. Thomas, and L. Zhang, Nat. Methods 10, 641 (2013).

${ }^{5}$ R. R. Harrison, in Proceedings of the IEEE Custom Integrated Circuits Conference (2007), pp. 115-122.

${ }^{6}$ A. Tarasov, W. Fu, O. Knopfmacher, J. Brunner, M. Calame, and C. Schönenberger, Appl. Phys. Lett. 98, 012114 (2011).

${ }^{7}$ E. K. C. Tee, A. Hölke, S. J. Pilkington, D. K. Pal, N. L. Yew, and W. A. W. Z. Abdin, IOSR J. Electr. Electron. Eng. 3, 35 (2012).

${ }^{8}$ E. A. Vittoz, IEEE J. Solid-State Circuits 18, 273 (1983).

${ }^{9}$ P. D. Batista and M. Mulato, Appl. Phys. Lett. 87, 143508 (2005).

${ }^{10}$ S. Chen and S.-L. Zhang, Anal. Chem. 83, 9546 (2011).

${ }^{11}$ S. M. Sze and K. K. Ng, Physics of Semiconductor Devices (John Wiley \& Sons, Hoboken, NJ, 2007), pp. 303-316.

${ }^{12}$ A. van der Ziel, Noise in Solid State Devices and Circuits (Wiley, New York, 1986).

${ }^{13}$ B. J. Baliga, Fundamentals of Power Semiconductor Devices (Springer, New York, NY, 2008), pp. 737-739.

${ }^{14}$ H. Yuan, H. C. Kwon, S. H. Yeom, D. H. Kwon, and S. W. Kang, Biosens. Bioelectron. 28, 434 (2011). 